\title{
Progress on Proving the Mass gap for Yang Mills and Gravity (Maybe it's already proven...)
}

\author{
Stephane H. Maes ${ }^{1}$
}

June 13, 2020

\begin{abstract}
:
Proving and constructing viable Yang Mills Gauge is a key concern for the Standard Model and an open problem. It has only be solved on lattices. Yet, gravity is not modeled in the Standard Model. We discuss that in a multi-fold universe where gravity emerges from entanglement effects, the spacetime is discrete (fractal with fractional dimensions, noncommutative and still Lorentz invariant). For any Lorentz invariant discrete spacetime, the lattice proofs and their lattice cell size independence completes the proof of the mass gap for Yang Mills Gauge theories. Continuous spacetime may or may not have a mass gap; but it does not matter if the real universe is discrete and Lorentz invariant.
\end{abstract}

The new preprint [1] proposes contributions to several open problems in physics like the reconciliation of General Relativity with Quantum Physics, explaining the origin of gravity proposed as emerging from quantum (EPR-

Einstein Podolsky Rosen) entanglement between particles, detailing contributions to dark matter and dark energy and explaining other Standard Model mysteries without requiring New Physics beyond the Standard Model other than the addition of gravity to the Standard Model Lagrangian. All this is achieved in s multi-fold universe that may well model our real universe, which remains to be validated.

With the proposed model of [1], spacetime and Physics are modeled from Planck scales to quantum and macroscopic scales and semi classical approaches appear valid till very small scales. In [1], it is argued that spacetime is discrete, with a random walk-based fractal structure, fractional and noncommutative at, and above Planck scales (with a 2-D behavior and Lorentz invariance preserved by random walks till the early moments of the universe). Spacetime results from past random walks of particles. Spacetime locations and particles can be modeled as microscopic blackholes (Schwarzschild for photons and spacetime coordinates, and metrics between Reisner Nordstrom [2] and Kerr Newman [3] for massive and possibly charged particles - the latter being possibly extremal). Although surprising, [1] recovers results consistent with other like [13], while also being able to justify the initial assumptions of black holes from the gravity or entanglement model. The resulting gravity model recovers General Relativity at larger scale, as a 4-D process, with massless gravity, but also with massive gravity components at very small scale that make gravity significant at these scales. Semi-classical models also work well till way smaller scales that usually expected.

Today, proving reconstruction and viability of Yang Mills Gauge theories is still an open problem. It is called the mass gap problem of Yang Mills Gauge theories[4]. A mass gap exists if there is a lower bound of the energy of any state which is orthogonal to the vacuum. The greatest such lower bound is the mass gap. If it exists for Yang Mills Gauge theory then Yang Mills theories can be reconstructed from an axiomatic model (instead of lattices or perturbative methods) and glueballs (particles formed only of gluons) can exist [5]. Proving the mass gap is important because Yang Mills gauge theories are behind the models of the strong interaction (QCD) and the Standard Model. Today, It is unsolved [4,12]. The impossibility, so far, to prove Yang Mills existence and stability, in $4-D$, is such a problem that a significant price $[6,7]$ is being offered for a rigorous resolution.

\footnotetext{
${ }^{1}$ shmaes.physics@gmail.com
} 
As explained for example in [4], proofs of the existence of a mass gap have been obtained on lattices [8,9]. [9] rather relies on strings and high $\mathrm{N}$ (in $\mathrm{SU}(\mathrm{N})$ ) symmetry groups. As superstrings are themselves not yet validated and as [8] is more straightforward lattice QCD, we prefer to rely on[8]. Its results are for lattices of fractions of femtometers and shows independence of the cell size around such scales.

As mentioned above, [1] shows that spacetime is discrete and can essentially be modeled with a lattice at such scales and below. The cell sizes are smaller, but the random walk skips nodes. So such a lattice or a few order of magnitude smaller would match. The approach in [1] also follows and discuss how it fulfills the QFT reconstruction axioms when at larger scales. Therefore, one can infer that a result à la [8] is actually nonbiased by the lattice and associated Gibbs effects.

In the framework of [1], [8], or a similar exercise on a lattice with one or to order of magnitude smaller cells, proves that the mass gap conjecture for Yang Mills Gauge field in 4-D. Of course, to complete the challenge, [1] must be validated as modeling our real universe. [1] proposed way to achieve this, e.g. by showing gravity-like fluctuation in entangled systems like superconductor or Qubits.

With this short paper, relying on [1] and [8], we have demonstrated a mass gap for Yang Mills Gauge theories in 4$D$, but only for a universe modeled by [1]: a multi-fold universe. [1] details arguments and ways to check its relationship with the real universe. Besides properties that can be experimentally verified (in the future because of the macroscopic weakness of gravity and gravity like effects for entangled systems), [1] shows how the multi-fold mechanisms and behaviors are in many aspects of in today's conventional physics, that, at times, anticipates the behaviors modeled of a multi-fold universe. In addition, [1] explains many results obtained in gravity, quantum mechanics, General Relativity, superstring theory, Loop Quantum Gravity and the AdS/CFT correspondence conjecture. All these works attempt to come up with models for the real universe. It is at least a good sign that [1] may provide an interesting model of the real universe.

Alternatively, if the reader is not yet convinced, our present paper has argued that it would suffice to show a derivation proving that our real spacetime is discrete (the fractal structure generated by random walk, fractional and noncommutative are less important as long that it has a Lorentz invariant behavior (and why)), at and above Planck scales up to fractions of $\mathrm{fm}$. Depending on the point of view, this argument closes the matter or provides a clear path to proving the mass gap for Yang Mills Gauge theories in 4-D: prove that [1] is the right model for the real universe or prove that the real universe is a discrete and Lorentz invariant spacetime at small scales, where general relativity can be recovered at larger scales. That is actually widely hypothesized to be the case. So far, the challenge so far has been the lack of a justification for such a discrete spacetime that would also be Lorentz invariant and evolve towards something appearing continuously 4-D spacetime, following GR, at large scales. [1] resolves the issues by justifying a random walk construction of spacetime that guarantees Lorentz invariance till the earliest moment of the universe and as a result fractal and noncommutative geometry that do the transition from 2-D dominant behavior to 4-D. That was in our view the missing link.

By the way, the microscopic black holes discussed in [1] are a way to justify the discrete aspects with recovery of GR. They result from the top down analysis of the effects of entanglement generating gravity like effects when multi-folds are involved, while multi-folds help explain EPR entanglement. They are not relevant to the mass gap problem. So a variation on [1] without them would do equally well.

Yes, this reasoning is probably not what the price organizer had in mind. Yet it is fair to ask if the hypothesis, asked to be proven, is provable in a continuous spacetime (it hasn't happened yet) or if it is even desirable, as we know that spacetime is discrete when gravity is involved $[1,10,11]$. So the continuous proof is probably less adequate for our reality that a proof on a suitable lattice or discrete spacetime.

[10] and [11] were not completing the proof by them-selves, as they only justified, respectively, non-commutativity or discreteness. The latter mentioned random sprinkles of point to justify Lorentz invariance but not why it would be random. [1] does. But these references are really why we stated earlier that, even if preferred left ignored, discreteness and Lorentz invariance are facts of our real universe; not just dreams of [1] and a few other 
researchers. Maybe it does not fit many superstring, QFT or other popular frameworks or formulations like for the Standard Model but hiding that aspects away does not make it go away. The challenge for a proof, or an proof of the existence of a mass gap in Yang Mills Gauge theory (in 4-D), acceptable to the Physics community, should be open to proofs on a discrete Lorentz invariant spacetime, if such a nature of spacetime is also suitably motivated. [1] presents strong arguments and enough examples that gravity, when added to the Standard Model or the standard Cosmology Model, impacts interestingly the outcome and helps resolves other challenge already. So maybe, it is time to consider a discrete reconstructive QFT model and a Standard Model on discrete spacetime. We say discrete spacetime and not lattice, to emphasize that the lattice is the end game, the real thing, not an interim simulation environment possibly prone to discrete biases and artefacts à la Gibbs.

We hope that this short analysis will motivate the Physics community to investigate how gravity and discrete Lorentz invariant spacetime are the, or at least a way to a proof relevant to our real universe instead of no proof at all, or a proof on a continuous spacetime that may not matters at the scales, involved for say QCD, where the special features of Yang Mills non abelian Gauge theories, like confinement, really come into play. After all, if the mass gap were proven, say in the context of superstrings, it would suffer from its own lack of any experimental validation (this is why we did not argue using [9], to avoid bringing these considerations also to our argument. Analyses and considerations like in [14], do not give us a way yet to accept string results in the multi-fold spacetime as proofs.). At least, [1] provides predictions and suggestions for its own falsifiability.

References: (most references come from popular science to make the discussion more approachable)

[1]: Stephane H. Maes, (2020) "Quantum Gravity Emergence from Entanglement in a Multi-Fold

Universe", viXra:2006.0088v1, (June 9, 2020).

[2]: https://en.wikipedia.org/wiki/Reissner\%E2\%80\%93Nordstr\%C3\%B6m metric

[3]: https://en.wikipedia.org/wiki/Kerr-Newman metric

[4]: https://en.wikipedia.org/wiki/Yang\%E2\%80\%93Mills existence and mass gap

[5]: https://en.wikipedia.org/wiki/Glueball

[6]: https://en.wikipedia.org/wiki/Millennium Prize Problems

[7]: http://www.claymath.org/sites/default/files/yangmills.pdf

[8]: Lucini, Biagio; Teper, Michael; Wenger, Urs (2004). "Glueballs and k-strings in SU(N) gauge theories : calculations with improved operators". Journal of High Energy Physics. 0406 (6): 012. arXiv:hep-lat/0404008.

[9]: Chen, Y.; Alexandru, A.; Dong, S. J.; Draper, T.; Horvath, I.; Lee, F. X.; Liu, K. F.; Mathur, N.; Morningstar, C.; Peardon, M.; Tamhankar, S.; Young, B. L.; Zhang, J. B. (2006). "Glueball Spectrum and Matrix Elements on Anisotropic Lattices". Physical Review D. 73 (1): 014516. arXiv:hep-lat/0510074.

[10]: S. Doplicher, K. Fredenhagen and J. E. Roberts, (1994), "Spacetime quantization induced by classical gravity", Phys. Rev. B 331 (1994) 33.

[11]: Hooft, Gerard 't, (2016), "How quantization of gravity leads to a discrete space-time", J. Phys.: Conf. Ser. 701 012014

[12]: https://www.claymath.org/sites/default/files/ym2.pdf

[13]: Burinskii, Alexander, (2008), "The Dirac-Kerr-Newman electron", arXiv:0507109v4

[14]: Stephane H Maes, (2020), " Dualities or Analogies between Superstrings and Multi-fold Universe ", viXra:2006.0178v1, https://shmaesphysics.wordpress.com/2020/06/14/dualities-or-analogies-betweensuperstrings-and-multi-fold-universes/, June 14, 2020. 